\title{
Effects of moderate, voluntary ethanol consumption on the rat and human gut microbiome
}

\author{
Kassi L. Kosnicki ${ }^{1}$, Jerrold C. Penprase ${ }^{2}$, Patricia Cintora ${ }^{2}$, Pedro J. Torres ${ }^{1}$, Greg L. \\ Harris ${ }^{1}$, Susan M. Brasser ${ }^{2, *}$, and Scott T. Kelley ${ }^{1,{ }^{*}, \dagger}$ \\ ${ }^{1}$ Department of Biology, San Diego State University, San Diego, CA, 92104 USA \\ ${ }^{2}$ Department of Psychology, San Diego State University, San Diego, CA, 92182 USA
}

\begin{abstract}
Many alcohol-induced health complications are directly attributable to the toxicity of alcohol or its metabolites, but another potential health impact of alcohol may be on the microbial communities of the human gut. Clear distinctions between healthy and diseased-state gut microbiota have been observed in subjects with metabolic diseases and recent studies suggest that chronic alcoholism is linked to gut microbiome dysbiosis. Here, we investigated the effects of moderate levels of alcohol consumption on the gut microbiome in both rats and humans. The gut microbiota of rats voluntarily consuming a $20 \%$ ethanol solution, on alternate days, were compared to a non-exposed control group to identify differential taxonomic and functional profiles. Gut microbial diversity profiles were determined using culture-independent amplification, next-generation sequencing and bioinformatic analysis of bacterial 16S ribosomal RNA gene sequence libraries. Our results showed that, compared to controls, ethanol-consuming rats experienced a significant decline in the biodiversity of their gut microbiomes, a state generally associated with dysbiosis. We also observed significant shifts in the overall diversity of the gut microbial communities and a dramatic change in the relative abundance of particular microbes, such as the Lactobacilli. We also compared our results to human fecal microbiome data collected as part of the citizen science American Gut Project. In contrast to the rat data, human drinkers had significantly higher gut microbial biodiversity than non-drinkers. However, we also observed that microbes that differed among the human subjects displayed similar trends in the rat model, including bacteria implicated in metabolic disease.
\end{abstract}

\section{Keywords}

alcohol; gut microbiome; metagenomics

\footnotetext{
${ }^{\dagger}$ Correspondence: Scott T. Kelley, Department of Biology, 5500 Campanile Drive, San Diego State University, San Diego, CA 92104-4614, USA, skelley@mail.sdsu.edu; Phone: +1 619206 8014; Fax: +1 6195945676.

These senior authors contributed equally to the project and manuscript.

The authors declare no conflict of interest.

Authors Contribution

STK, SMB, GLH and KLK were responsible for the study concept and design. JCP and PC contributed to the acquisition of animal data. PJT assisted QIIME and R data analysis and interpretation. KLK, STK and SMB were responsible for data analysis and interpretation of findings and drafted the manuscript. GLH provided critical revision of the manuscript for important intellectual content. All authors critically reviewed content and approved final version for publication.
} 


\section{Introduction}

Archaeologists trace the brewing of alcoholic beverages by humans back to the Neolithic, indicating that alcohol consumption has been part of human culture for millennia (Cordain et al., 2005; Dietrich et al., 2012). While many studies have indicated that modest daily intake of alcohol may have health benefits (Collins et al., 2009, for review), excessive alcohol consumption is known to result in a variety of serious health problems, including alcoholic liver disease (Mutlu et al., 2009; Mutlu et al., 2012), cirrhosis (Bajaj et al., 2014; Chen et al., 2011; Chen et al., 2015), cancers (Room et al., 2005) and neurologic impairment (Cargiulo, 2007). Excessive drinking rates have been on the rise in the United States and other countries (Dwyer-Lindgren et al., 2015), which has also led to an increase in alcoholinduced deaths, including homicides and alcohol-related accidents according to the Centers for Disease Control and Prevention (CDC).

Many alcohol-induced health complications and diseases are attributed to the direct toxicity of alcohol or its metabolites (i.e., acetaldehyde). Another potential and related health impact of alcohol ingestion may be on the microbial communities that comprise the human gut microbiome. Alteration of the gut microbiota has been observed in many disease states, such as irritable bowel syndrome (Carroll et al., 2011; Hold et al., 2014), cancer (Schwabe \& Jobin, 2013), polycystic ovary syndrome (Kelley et al., 2016), and even autism (Mulle et al., 2013). In a dysbiotic state, gut bacterial species normally present at low abundance in the gut come to dominate the microbial community (Keshavarzian et al., 2010; Mutlu et al., 2009; Yan et al., 2011). For instance, in a study comparing a healthy control group to alcoholics with and without liver disease, the abundance of Bacteroidetes in the gut microbiome of alcoholics was significantly lower than in the healthy controls, while Proteobacteria abundances were significantly higher (Mutlu et al., 2012). While the importance of 'bacterial overgrowth' as cause or effect in gut dysbiosis remains a topic of intense investigation, evidence is accumulating that such overgrowth can critically modify gene regulation in the host gut epithelium (Yan et al., 2011), alter immune response and gut permeability (Edelblum et al., 2018), and even induce cancer (Yu et al., 2017).

Similar abundance trends were observed in the fecal microbiota of subjects with alcoholrelated cirrhosis or hepatitis B (Chen et al., 2011). In the gut microbiota of mice, chronic ethanol exposure resulted in similar taxonomic reductions (Bull-Otterson et al., 2013). In the human gut, health-associated bacteria, such as Lachnospiraceae, Ruminococcaceae, and Lactobacillus spp. abundances are typically reduced in alcohol-dependent patients and those with cirrhosis while abundances of disease-associated bacteria belonging to the Enterobacteriaceae, Streptococcaceae, Prevotellaceae, Veillonellaceae, Bacteroidaceae, and the Clostridium spp. increase (Bajaj et al., 2014; Chen et al., 2011; Leclercq et al., 2014). These shifts and an overgrowth of bacteria in the gut have also been associated with increased intestinal permeability, contributing to increased endotoxin levels (Hartmann et al., 2015; Segawa et al., 2008) and the pathogenesis of alcohol-related diseases (Engen et al., 2015).

The analysis of the gut microbiota has been studied primarily in subjects with excessive alcohol exposure or alcohol-related diseases, however little is known regarding the effects of 
moderate levels of ethanol consumption on the gut microbiota. Moderate or "low-risk" drinking is defined by the National Institute on Alcohol Abuse and Alcoholism as no more than 7 drinks/week for women or 14 drinks/week for men, at non-binge levels (i.e., $<0.08$ $\mathrm{g} / \mathrm{dl}$ blood alcohol concentration; NIAAA, 2018). Various epidemiological studies have reported the effects of moderate ethanol consumption in relation to health/disease risk. For instance, the risk of breast cancer in women has been reported to increase when ethanol is consumed in moderation, as alcohol may act as a weak carcinogen and promote tumor growth (Brooks \& Zakhari, 2013). Several other studies have indicated health benefits associated with moderate drinking, including decreased rates of cardiovascular disease and reduced risk for dementia, type 2 diabetes, and osteoporosis (Collins et al., 2009). However, the benefits and risks of moderate ethanol consumption remain controversial, as a recent review of epidemiological studies reporting beneficial outcomes has shown that a misclassification of drinkers may have contributed to a bias in mortality risk among groups (Stockwell et al., 2016).

Here, we used culture-independent next-generation sequencing (NGS) and bioinformatic analysis to determine how moderate, voluntary alcohol consumption affects gut microbial diversity in an outbred Wistar rat model. We also compared our results to human data collected as part of the American Gut Project (AGP), a crowd-sourced initiative with over 6,000 contributing citizen scientists. Our results showed significant time-dependent changes to community-wide biodiversity of the rat gut ecosystem, with specific changes in bacterial genera known to be directly involved in metabolizing breakdown products of ethanol in the gut. We also found participants in the AGP who self-reported weekly or daily alcohol intake had significantly different microbial diversity compared to non-drinkers.

\section{Methods}

\subsection{Rat Model}

2.1.1 Experimental Design and Sample Collection-All procedures were approved by the IACUC at San Diego State University and were in accordance with NIH guidelines. At 109 days of age, male Wistar rats $(n=24 ; 503.0 \pm 10.9 \mathrm{~g}$, Harlan Laboratories, Placentia, CA, USA) were randomly assigned to two groups ( $n=12$ /group), control and ethanoltreated. All rats were fed a regular chow diet (LabDiet, St. Louis, MO, USA), ad libitum, and were housed in individual tub cages in a vivarium that maintained a 12:12 hr light/dark cycle and an ambient temperature of $\sim 23^{\circ} \mathrm{C}$. Rats were exposed for 13 weeks to either a $20 \%$ ethanol intermittent-access drinking paradigm (Simms et al., 2008) or were given access to water alone as their fluid source (non ethanol-exposed control). This alcohol exposure paradigm has been shown to result in blood alcohol concentrations averaging $\sim 30-50 \mathrm{mg} / \mathrm{dl}$ in the Wistar strain when measured 30-120 min into a typical drinking session (Cippitelli et al., 2012; Simms et al., 2008). Animals were initially acclimated to testing procedures for 7 days during which they received free access to food and water only. Following the acclimation period, ethanol-exposed rats began 22-hr intake sessions involving voluntary access to a $20 \%$ (v/v) ethanol solution vs. water, alternating with $22-\mathrm{hr}$ abstinence periods involving voluntary access to water only (45 ethanol drinking sessions total). The position (right or left) of ethanol and water bottles on each cage was rotated for successive ethanol 
sessions to control for drinking side preferences. The control group was given voluntary access to two water bottles on corresponding sides of the cage during the entire duration of the chronic exposure period. All bottles were weighed to the nearest gram at the start and end of each session and replenished with fresh fluids daily, and body weights of all rats were measured every $48 \mathrm{hr}$ before the start of each ethanol session. Fecal samples were collected from the rats prior to treatment at baseline (Time 0), then at 4-week intervals, with the final sample collected after the last ethanol exposure session (12 weeks, 6 days). When reporting time-dependent findings, we refer to this final collection as the 12-week mark. Fecal samples were frozen immediately after collection and stored at $-80^{\circ} \mathrm{C}$ for subsequent analysis.

2.1.2 DNA Extraction-Isolation of purified DNA was performed using MoBio PowerSoil ${ }^{\circledR}$ DNA Extraction Kit (MO BIO Laboratories, Inc., Carlsbad, CA, USA) following the manufacturer's instructions. DNA concentrations were determined using a Nanodrop 2000 (Thermo Fisher Scientific, Waltham, MA) and gel electrophoresis.

2.1.3 Next Generation Sequencing (NGS) of 16S rRNA Genes-The bacterial V4 hypervariable region of the $16 \mathrm{~S}$ ribosomal RNA sequence was PCR amplified from isolated DNA using "universal" $16 \mathrm{~S}$ primers ( $515 \mathrm{~F}$ and $806 \mathrm{R})$. Reverse primers contained a unique 12-base pair Golay barcode that was incorporated into the PCR amplicons (Fierer et al., 2008). Amplicons were sent to The Scripps Research Institutes NGS core facility for cleaning using DNA Clean \& Concentrator ${ }^{\mathrm{TM}}-25$ columns (Zymo, Irvine, CA), quantification using a Qubit Fluorometer (Life Technologies, Thermo Fisher Scientific), and pooling. Pooled PCR products were size selected on a $2 \%$ agarose gel (290-350bp) and purified using a Zymoclean ${ }^{\mathrm{TM}} \mathrm{Gel}$ Dna recovery kit to prepare sequencing libraries following the recommended Illumina protocol involving end repair, A-tailing and adapter ligation. The library was then amplified for 12 cycles using the HiFi Polymerase (Kapa Biosystems, Wilmington, MA). The purified DNA library was quantitated and denatured in $0.1 \mathrm{~N} \mathrm{NaOH}$ and diluted to a final concentration of $5 \mathrm{pM}$. Sequencing was performed using a 2x300 kit (version 3) on the Illumina MiSeq ${ }^{\circledR}$ system (Illumina, Inc., San Diego, CA, USA) along with 4 pM PhiX control library. Raw sequence reads were submitted to the Sequence Read Archive (SRA; Accession number: PRJNA417868) as an NCBI BioProject (ID: 417868) accessible at https://www.ncbi.nlm.nih.gov/bioproject/PRJNA417868.

2.1.4 16S rRNA Taxonomic Diversity Analysis-Sequence data were analyzed using the pipeline, Quantitative Insights Into Microbial Ecology (QIIME version MacQIIME 1.9.1-20150604; Caporaso et al., 2010). Forward and reverse reads were joined, demultiplexed, and quality filtered using the QIIME default filters in the split_libraries.py script. Sequences were clustered with uclust (Edgar, 2010) using a de novo operational taxonomic unit (OTU) picking method (pick_denovo_otus.py). Reads were aligned to the Greengenes Core reference alignment (DeSantis et al., 2006) using PyNAST (Caporaso et al., 2010). Chimeric screening was performed using the usearch61 (Edgar, 2010) method from the identify_chimeric_sequences.py script. OTU assignment was performed by comparing sequences to the Greengenes reference database (McDonald et al., 2012) with a 97\% pairwise identity cutoff using the RDP Classifier method (Wang et al., 2007). 
Sequences found in low abundance were filtered using the filter_otus_from_otu_table.py. Low abundance sequences were classified as those being present in less than $25 \%$ of the samples. Remaining sequences were then aligned using PyNast (Caporaso et al., 2010). Additional OTU tables, excluding time zero samples were generated to observe changes in diversity between control and ethanol-treated rats. Shannon index, Chao1, Faith's Phylogenetic Diversity, and observed species (observed OTUs) measures were calculated using the alpha_diversity.py script. Beta diversity was computed and visualized using unweighted and weighted UniFrac (Lozupone \& Knight, 2005) distances with the beta_diversity_through_plots.py script. See Supplemental Material for details on statistical analysis of Rat and Human model data.

\subsection{Human Model}

2.2.1 Human microbiome data from the American Gut Project-Data for analysis of the relationship between alcohol consumption and human gut microbial diversity came from the American Gut Project (www.americangut.org). This project generated microbial community sequencing data from more than 6,000 fecal samples donated by citizen scientists across the United States. Specifically, we used a pre-generated OTU (biom) table that included the abundances of microbial taxa for every sample. Details on the sample collection, preparation, sequencing and bioinformatics analysis can be found at http:// www.earthmicrobiome.org/. These samples were provided with metadata including personal information such as age, weight, dietary habits, and alcohol consumption. Frequency of alcohol consumption was self-reported via a survey however precise estimates of daily alcohol intake were not available. The OTU biom table, rarified to 10,000 sequences per sample, and the metadata file were downloaded from the AGP website (ftp:// ftp.microbio.me/AmericanGut/rounds-1-22/07-taxa/100nt/). Shannon index, Chao1, Faith's Phylogenetic Diversity, and observed species (observed OTUs) measures, UniFrac distances and relative abundances of taxa had all been pre-calculated as part of the AGP. Links to AGP data files and metadata are included as Supplemental Material.

2.2.2 Sample Selection and Exclusions-The full data set included 7183 samples with relative abundance estimates for 1982 bacterial taxa. These samples were swabbed from three body regions: skin, fecal, and oral. All samples were excluded except for fecal samples (total=6284), which were divided into three groups based on self-reported drinking habits. These groups were determined by the "ALCOHOL_FREQUENCY" parameter included in the metadata. Those who reported never drinking ("non-drinkers") totaled 1495 samples. The second subset included 1260 samples from individuals who reported drinking 3-5 times/week ("regular drinkers") and the final group including 692 samples from individuals who reported daily alcohol consumption ("daily drinkers"). The rest of the samples had unknown levels of consumption and were excluded from the analysis.

\section{Results}

\subsection{Ethanol Intake-Rat Model}

Rat ethanol intake over the course of the study is represented in Figure 1 and described in Supplemental Material. 


\subsection{Rat Gut Microbiome}

\subsubsection{Alcohol Consumption Induces a Shift in the Gut Microbial Community in}

Rats-We obtained an average of $\sim 17,000$ sequences per sample, post-quality filtering and chimera checking, from 89 rat microbiome samples. De novo clustering produced a total of 904 OTUs across all samples. No significant shifts were detected at the phylum level in any of the bacteria amongst time points in the ethanol-treated rats. In the control group, the relative abundances of the Proteobacteria and Deferribacteres phyla changed over time (Figure 2), though only the Lentisphaerae abundances were significantly different between treatments (Bonferroni, $\mathrm{p}=0.0109$ ). Principle Coordinate Analysis $(\mathrm{PCoA})$ plots of both the weighted and unweighted UniFrac distances found in the overall gut microbiota between treatment and control groups, a pattern confirmed via a non-parametric Analysis of Similarities, an ANOVA like test that uses a ranked dissimilarity matrix (Figure 3; ANOSIM weighted UniFrac, $\mathrm{p}=0.033$; unweighted UniFrac, $\mathrm{p}=0.004$ ). UniFrac is a metric of beta diversity that incorporates phylogenetic information when estimating the distances between all pairs of microbial communities, in this case bacterial genera of each gut sample.

Bacterial samples that are more similar to one another will have a smaller distance, while samples that are dissimilar will have a larger distance. Unweighted UniFrac values only incorporate the phylogenetic relationships among genera, while weighted UniFrac "weights" the phylogenetic information by the abundance of each genus in the community (see Louzopone \& Knight, 2005). There were no significant differences in the gut microbiota of the control group over time (data not shown), but PCoA plots of weighted UniFrac distances over the 12-week period showed differential gut microbiota in the ethanol group (Figure 3C$\mathrm{D}$; ANOSIM weighted UniFrac, $\mathrm{p}=0.04399$, unweighted UniFrac, $\mathrm{p}=0.026$ ). Comparisons of alpha-diversity (within sample microbial diversity) found that rats in the control group had higher average species richness and phylogenetic diversity than the ethanol group (Figure 4). Both control and ethanol groups displayed a similar positive trend in gut biodiversity, but the trend was only significant in the control group (Figure 5).

3.2.2 Comparisons of taxonomic abundance-Overall abundance at the phylum level indicated that $\sim 97 \%$ of data clustered to either Firmicutes or Bacteroidetes, which is typically observed in both rodent and human gut microbiomes (Eckburg et al., 2005; Turnbaugh et al., 2006). The clearest differences in organismal diversity between control and treatment groups were observed at the level of specific genera, namely Lactobacillus and Parabacteroides. Figure 6 shows the average abundance of Lactobacillus, which was significantly lower in ethanol-treated rats. The abundance of Parabacteroides, on the other hand, was significantly greater in ethanol-treated rats compared to controls (Figure 7). In the control group, abundances of Lactobacillus remained relatively constant over time, while in ethanol-treated rats, we observed a distinct decrease by the fourth week of treatment (Figure 6). Other OTUs whose relative abundances differed collectively over the course of the study between treatment groups (Kruskal-Wallis; $\mathrm{p}<0.05$ ) included members of the Lentisphaerae (family Victivallaceae), the Methanosphaera (Euryarchaeota), 6 OTUs belonging to the Bacteroidetes and Firmicutes, and members of the genus Phascolarctobacterium (Figure 8; Table 1). 


\subsection{Human Gut Microbiome}

\subsubsection{Correlation of Alcohol Consumption and Gut Microbial Community}

Composition in Humans-Both weighted and unweighted UniFrac analyses were used to compare the diversity of the gut microbiome, at the genera level, between classifications of drinkers. PCoA plots of both the weighted and unweighted UniFrac distances were used to compare regular drinkers (3-5 times a week) and daily drinkers to non-drinkers (data not shown). While the comparisons showed no clear visual discrimination of the microbial communities, an ANOSIM test found a significant difference in the unweighted UniFrac distances (weighted UniFrac, $\mathrm{p}=0.992$; unweighted UniFrac, $\mathrm{p}=0.0280$ ). ANOSIM results comparing daily drinkers to non-drinkers showed no significant difference in microbial community composition with either weighted or unweighted UniFrac distances.

\subsubsection{Association of Alpha Diversity and Alcohol Consumption in Humans-In} contrast to the rat model, analysis of alpha diversity determined that self-reported nondrinkers had significantly lower species richness and phylogenetic diversity compared to regular and daily drinkers (Figure 9). Diversity was significantly greater in the gut microbiome of both "regular" and "daily" drinkers compared to non-drinkers, but no difference was observed between "regular" and "daily" groups. Table 2 summarizes differences in alpha diversity between control and ethanol-drinking subjects in both models. The two measures, Faith's Phylogenetic Diversity and Chao1, differed significantly between non-drinkers and drinkers (Figure 9). Because microbial composition was similar in both drinking groups, data from "daily" and "regular" drinkers was subsequently combined to represent the ethanol group for comparison against those that never drank.

3.3.3 Comparison of Human and Rat Gut Microbiomes-Because their abundances differed between treatments in the rat model, Lactobacillus and Parabacteroides abundances were compared between drinking and non-drinking individuals to identify if there were similar trends between models. Lactobacillus abundances did not differ between drinkers and non-drinkers and Parabacteroides abundances were greater in drinking individuals, consistent with the rat model. In addition to Parabacteroides, the Kruskal-wallis test identified 21 OTUs whose abundances differed $(\mathrm{p}<0.05)$ between drinkers and non-drinkers, with the top three OTUs identified as Barnesiellaceae, RF32, and Eggerthella. The abundances of these OTUs were compared to the rat model to identify any similar trends (Table 1). Most of the OTU abundances between treatment were similar between models, with the exception of Coriobaceteriaceae- and Dehalobactium-identified OTUs as well as four undetected OTUs identified as cc15, Eggerthella, Lachnobacterium, and Odoribacter.

\section{Discussion}

Moderate levels of voluntary alcohol intake had a significant impact on Wistar rat gut microbial diversity. Over the course of the experiment, rats exposed to the $20 \%$ ethanol intermittent-access paradigm gradually increased both preference for and intake of ethanol, with final average intake levels of $\sim 5 \mathrm{~g} / \mathrm{kg} /$ day (Figure 1). This drinking paradigm has been shown to result in blood alcohol concentrations (BACs) averaging $\sim 30-50 \mathrm{mg} / \mathrm{dl}$ in the Wistar strain when measured 30-120 min into a standard drinking session, when intake 
levels typically peak (Cippitelli et al., 2012; Simms et al., 2008). The latter BAC approximately corresponds to an acute $0.5 \mathrm{~g} / \mathrm{kg}$ dose in human drinkers (Brasser et al., 2004), or that normally resulting from 1-2 standard drinks. Bioinformatics analysis of the gut microbial communities over the course of the experiment found clear separation of the beta (between sample) diversity between control and ethanol-fed animals (Figures 2 and 3). The effect was more pronounced with the weighted UniFrac distances compared with the unweighted, though significant separation was observed with both distance measures. Since the unweighted UniFrac distances are only based on the evolutionary diversity of the bacterial community, the stronger explanatory power of the weighted UniFrac suggests that the largest impact of ethanol resulted from shifts in the relative abundance of taxa common to both control and ethanol-treated rats.

We also determined a significant difference in the alpha (within sample) microbial diversity between control and ethanol-treated groups. Average species richness (the number of different type of species; chao1) and phylogenetic diversity (Faith's PD) were both significantly lower in ethanol-treated rats compared with control (Figure 4). A regression analysis of alpha diversity measured over time found a significant positive correlation between alpha diversity measurements in control rats, while no significant correlation was found in ethanol-treated rats (Figure 5). In healthy humans and rodents, alpha diversity tends to increase with age (Yatsunenko et al., 2012). On the other hand, decreased alpha diversity is typically found in dysbiotic states, including different forms of bowel disease, intestinal infections, obesity, polycystic ovary syndrome and other diseases with metabolic phenotypes (Carroll et al., 2011; Chiu et al., 2014; Hold et al., 2014; Kelley et al., 2016). Interestingly, high-fat diets, obesity, and alcoholism have also all been linked to increased serum endotoxin levels (Tilg \& Kaser, 2011). Specifically, these studies have connected altered microbial composition to intestinal permeability and bacterial products, principally LPS, leaking across the intestinal epithelium.

In terms of changes to specific microbial taxa, the most pronounced difference between control and treatment groups was in the relative abundance of Lactobacillus. The average relative Lactobacillus abundance over the course of the experiment was significantly lower in treatment than control animals (Figure 6). This difference was observable after only 4 weeks, and the Lactobacillus abundance remained low throughout the rest of the study, while the Lactobacillus abundance remained constant in controls. These data are consistent with prior findings of strongly suppressed Lactobacillus abundances in C57/B6 mice following 3week intragastric administration of high ethanol doses (up to $30.9 \mathrm{~g} / \mathrm{kg} /$ day) to induce alcoholic liver disease (ALD; Yan et al., 2011). Our results indicate that declines in Lactobacillus may be observed at much lower, moderate levels of voluntary alcohol consumption, in animals maintained on a standard diet that does not induce ALD. Other bacterial taxa also decreased over time in the treatment group compared with the control, including members of the Peptostreptococcaceae and Turicibacter (Figure 6), while other bacterial groups were higher overall in the treatment cohort than in controls, including members of the Parabacteroides, Barnesiellaceae and Bacteroides (Figure 7). A relative decrease in Firmicutes and increase in Bacteroidetes has also been observed at higher levels of ethanol exposure in mice (Yan et al., 2011), although the significance of shifts in specific microbial taxa are not yet well-characterized. (It should be noted that the differences in the 
relative abundances observed in Figures 6 and 7 typically resulted from a differential decrease in one treatment group relative to the other.) Other groups whose relative abundance differed in ethanol-treated rats include members of the genus Phascolarctobacterium and, interestingly we discovered that a genus of Archaea that happened to be amplified by our primers, Methanosphaera, also increased in the treatment group (Figure 8).

Of these microbial taxa, the connection between ethanol and the gut microbiome has been best characterized for Lactobacillus. The gut microbiome converts alcohol to acetaldehyde, and Lactobacillus help convert acetaldehyde to acetate. Acetaldehyde has been shown to disrupt the epithelial barrier by interfering with the tight junctions between the epithelial layers, which leads to increased permeability and leakage of bacteria and lipopolysaccharides (LPS) (aka., endotoxins) across the membrane resulting in endotoxemia. Thus, Lactobacillus acts to protect the gut lining and appears to prevent or reduce endotoxemia. Indeed, numerous studies have reported success in using Lactobacillus as a probiotic in humans and rodent models to prevent or reduce alcohol-induced dysbiosis, intestinal hyperpermeability, endotoxemia, and associated liver injury (Bajaj et al., 2014; Bull-Otterson et al., 2013; Mutlu et al., 2009; Nanji et al., 1994; Wang et al., 2011, 2012).

While decline of Lactobacillus relative abundance in ethanol-treated rats was significant and consistent over time, the reasons for this decline are less obvious. In general, Lactobacilli grow very well under high ethanol conditions. One suggestion is that alcohol consumption may change the gut $\mathrm{pH}$ enough to make the gut less habitable for Lactobacillus. Other possibilities include resource competition with other microbes, or possible changes in the host that disfavor the growth of these organisms. Chronic ethanol feeding in mice has been reported to result in increased fecal $\mathrm{pH}$ (Bull-Otterson et al., 2013), which is reduced by Lactobacillus supplementation.

In addition to the changes in relative bacterial abundances, we also found a significant difference in the relative abundance of the one Archaea that we determined,

Methanosphaera, a genus that has been well studied in the gut ecosystem (Figure 8). As one of the few mammalian Archaea gut commensals, these organisms are well known as producers of intestinal (methane) gas by using hydrogen gas to reduce methanol. However, what is less well appreciated is the fact that these organisms require acetate and $\mathrm{CO}_{2}$ as carbon sources for growth. Moreover, in some animals, strains of Methanosphaera are known to derive energy directly from ethanol. Thus, the increase in the relative abundance of Methanosphaera could coincide with an increase in ethanol and, subsequently, acetate production, though it is unclear what microbes would produce the acetate given the lowered relative abundance of Lactobacillus.

\section{Comparisons to human gut microbiome}

The release of gut (fecal) microbiome data from the American Gut Project (AGP) allowed us the opportunity to compare/contrast the effects of moderate voluntary alcohol consumption on gut microbial diversity in an experimental rat model to that of humans who self-reported different frequencies of alcohol consumption. Obviously, there were many differences between these treatment models making the comparison far from ideal. For example, (1) we 
could control and precisely measure intake in the rats, but not humans (the AGP dataset included frequency and type but not quantity of alcohol consumed), (2) self-reports can be inaccurate, (3) we only had one time point measure for the humans, and (4) the human participants differed in age, genetics, and many environmental factors.

Nevertheless, the large sample size of the AGP cohort meant we could use thousands of samples in the analysis, and such large sample sizes can allow patterns to be seen over the noise of other factors. Indeed, we found significant differences in the community-wide composition between non-drinkers ("Control") and regular/daily drinkers ("Ethanol") in both alpha and beta diversity. Unlike in the rats, the alpha diversity of regular or daily drinkers was significantly higher than non-drinkers (Figure 9). Since higher alpha diversity in the human gut is correlated with greater health, it is possible that the level of drinking in these AGP participants may have had a beneficial effect. We note that approximately half (48\%) of all drinkers in the AGP sample reported consuming red wine (Supplemental Table S2). Moderate daily consumption of red wine in healthy volunteers has been reported to induce potentially favorable changes in the gut microbiome possibly due to prebiotic benefits associated with the high polyphenol content of red wine (Queipo-Ortuno et al., 2012). By contrast, excessive alcohol consumption produces a dysbiotic state in a proportion of alcoholics (cirrhotic or with or without liver disease) that is characterized by increases in potentially pathogenic gram-negative bacteria including Proteobacteria, and decreased Bacteriodetes abundances (Bode et al., 1984; Chen et al., 2011; Mutlu et al., 2012).

It should also be noted, however, that the human participants had likely been drinking alcohol for many years (mean age of combined drinkers $=51.34$ years) while the rats only consumed ethanol for 12 weeks. It is possible that humans also experience an initial reduction in gut microbial diversity when they begin consuming alcohol, most often in the teens and early 20s. Indeed, we noticed a slight upward trend in alpha diversity measures by week 12 of the experiment in the rat model, which may have continued if the experiment had been extended (Figure 5). This also seemed true of some of the other bacteria apart from the Lactobacillus (Figure 6). Alternatively, the human gut and its microbial ecosystem may have evolved to better tolerate regular ethanol consumption. As mentioned in the introduction, humans have been brewing and drinking alcoholic beverages for millennia and our gut bacteria and large intestine may have evolved to tolerate both regular and highly variable levels of alcohol consumption.

Unweighted UniFrac measures also indicated a significant (ANOSIM; $\mathrm{p}=0.028$ ) differentiation in non-drinkers vs. regular drinkers, though we did not see a community-wide difference in the weighted UniFrac metric (data not shown). Despite the differences in alpha diversity, when comparing between treatments we observed that many of the OTUs that differ in humans displayed similar trends in the rat model (Table 1). For instance, Parabacteroides, Adlercreutzia, Barnesiellaceae, Butyricimonas, Clostridiales, Oxalobacter, Paraprevotella, Phascolarctobacterium, RF32, Ruminococcaceae, Sutterella, S24-7, and YS2-identified OTU abundances were higher in both rat and human ethanol-treated groups. Peptococcus, Clostridiaceae, and Lactobacillus-identified OTU abundances were lower in both rat and human ethanol drinkers. The decrease we observed in Lactobacillus abundances may foreshadow how heavier or continued ethanol drinking could affect rat and human 
drinking groups. As noted previously, significant reductions in Lactobacillus may serve as a precursor for increased gut permeability and endotoxemia.

Though similar trends were observed when comparing mean abundances between treatments and models, there were some clear differences. For instance, the most affected OTUs, in response to ethanol consumption, differed between models. In rats, Lactobacillus (Firmicutes) and Parabacteroides (Bacteroidetes) OTUs experienced the greatest shifts. The top three OTUs that differed between human drinkers and non-drinkers were identified as Barnesiellaceae (Bacteroidetes), RF32 (Proteobacteria), and Eggerthella (Coriobacteriaceae). When comparing OTUs that differed between treatment in rats to those that differed in humans there were many that were altered in humans but not rats (Table 1). Of those OTUs that did show overlap, all except Coriobacteraceae and Dehalobacterium had similar trends between models. Butyricimonas species are butyrate-producing bacteria that are important to colon health in humans (Barcenilla et al., 2000). In humans, Butyricimonas abundances were significantly higher in drinkers compared with non-drinkers.

In summary, the decline of gut microbiome biodiversity and the shift in overall microbial composition observed in our study suggests that the rat microbiome may be negatively affected by short-term moderate levels of consumption. In addition, Lactobacillus abundances were significantly decreased, potentially creating a vulnerable epithelial barrier and a potential for endotoxemia. Future studies monitoring these levels in blood would further clarify the relationship between the gut microbiota and the immune system. Additionally, it would be interesting to extend the study over a longer timeframe of ethanol exposure in the rat model. For example, by week 12 the relative abundances of some bacterial taxa which were initially depleted seemed to rebound (Figures 6 and 7). With additional sample collections, we may observe recovery in gut microbial diversity over time. In the future, it would also be important to measure levels of acetaldehyde and gut barrier function in the rat model. Finally, a study looking at gut microbial diversity and permeability in humans as they begin to consume alcohol for the first time (e.g., adolescence/early adulthood) could help determine whether the human gut microbiome experiences a similar initial reduction in biodiversity that later adapts with continued ethanol exposure.

\section{Supplementary Material}

Refer to Web version on PubMed Central for supplementary material.

\section{Acknowledgments}

This research was supported by NIH Grant AA023291 (SMB).

\section{References}

Bajaj, Jasmohan S; Heuman, Douglas M; Hylemon, Phillip B; Sanyal, Arun J; White, Melanie B; Monteith, Pamela; Noble, Nicole A; , et al. 2014; Altered Profile of Human Gut Microbiome Is Associated with Cirrhosis and Its Complications. Journal of Hepatology. 60(5):940-47. DOI: 10.1016/j.jhep.2013.12.019 [PubMed: 24374295]

Barcenilla A, Pryde SE, Martin JC, Duncan SH, Stewart CS, Henderson C, Flint HJ. 2000; Phylogenetic Relationships of Butyrate-Producing Bacteria from the Human Gut. Applied and 
Environmental Microbiology. 66(4):1654-61. DOI: 10.1128/AEM.66.4.1654-1661.2000 [PubMed: 10742256]

Bode JC, Bode C, Heidelbach R, Dürr HK, Martini GA. 1984; Jejunal microflora in patients with chronic alcohol abuse. Hepatogastroenterology. 31:30-4. [PubMed: 6698486]

Brasser SM, McCaul ME, Houtsmuller EJ. 2004; Alcohol effects during acamprosate treatment: A dose-response study in humans. Alcohol Clin Exp Res. 28:1074-1083. DOI: 10.1097/01.alc. 0000130802.07692.29 [PubMed: 15252294]

Brooks, Philip J; Zakhari, Samir. 2013; Moderate Alcohol Consumption and Breast Cancer in Women: From Epidemiology to Mechanisms and Interventions. Alcoholism: Clinical and Experimental Research. 37(1):23-30. DOI: 10.1111/j.1530-0277.2012.01888.x

Bull-Otterson, Lara; Feng, Wenke; Kirpich, Irina; Wang, Yuhua; Qin, Xiang; Liu, Yanlong; Gobejishvili, Leila; , et al. 2013; Metagenomic Analyses of Alcohol Induced Pathogenic Alterations in the Intestinal Microbiome and the Effect of Lactobacillus Rhamnosus GG Treatment. PloS One. 8(1):e53028.doi: 10.1371/journal.pone.0053028 [PubMed: 23326376]

Caporaso, J Gregory; Bittinger, Kyle; Bushman, Frederic D; Desantis, Todd Z; Andersen, Gary L; Knight, Rob. 2010; PyNAST: A Flexible Tool for Aligning Sequences to a Template Alignment. Bioinformatics. 26(2):266-67. DOI: 10.1093/bioinformatics/btp636 [PubMed: 19914921]

Caporaso, J Gregory; Kuczynski, Justin; Stombaugh, Jesse; Bittinger, Kyle; Bushman, Frederic D; Costello, Elizabeth K; Fierer, Noah; , et al. 2010; QIIME Allows Analysis of High-Throughput Community Sequencing Data. Nature Methods. 7(5):335-36. DOI: 10.1038/nmeth.f.303 [PubMed: 20383131]

Cargiulo, Thomas. 2007; Understanding the Health Impact of Alcohol Dependence. In. American Journal of Health-System Pharmacy. 64doi: 10.2146/ajhp060647

Carroll, Ian M; Ringel-Kulka, Tamar; Keku, Temitope O; Chang, Young-Hyo; Packey, Christopher D; Sartor, R Balfour; Ringel, Yehuda. 2011; Molecular Analysis of the Luminal- and MucosalAssociated Intestinal Microbiota in Diarrhea-Predominant Irritable Bowel Syndrome. American Journal of Physiology. Gastrointestinal and Liver Physiology. 301(5):G799-807. DOI: 10.1152/ ajpgi.00154.2011 [PubMed: 21737778]

Chen, Peng; Miyamoto, Yukiko; Mazagova, Magdalena; Lee, Kuei Chuan; Eckmann, Lars; Schnabl, Bernd. 2015; Microbiota Protects Mice Against Acute Alcohol-Induced Liver Injury. Alcoholism: Clinical and Experimental Research. 39(12):2313-23. DOI: 10.1111/acer.12900

Chen, Yanfei; Yang, Fengling; Lu, Haifeng; Wang, Baohong; Chen, Yunbo; Lei, Dajiang; Wang, Yuezhu; Zhu, Baoli; Li, Lanjuan. 2011; Characterization of Fecal Microbial Communities in Patients with Liver Cirrhosis. Hepatology. 54(2):562-72. DOI: 10.1002/hep.24423 [PubMed: 21574172]

Chiu, Chih Min; Huang, Wei Chih; Weng, Shun Long; Tseng, Han Chi; Liang, Chao; Wang, Wei Chi; Yang, Ting; , et al. 2014; Systematic Analysis of the Association between Gut Flora and Obesity through High-Throughput Sequencing and Bioinformatics Approaches. BioMed Research International. doi: 10.1155/2014/906168

Cippitelli, Andrea; Damadzic, Ruslan; Singley, Erick; Thorsell, Annika; Ciccocioppo, Roberto; Eskay, Robert L; Heilig, Markus. 2012; Pharmacological Blockade of Corticotropin-Releasing Hormone Receptor 1 (CRH1R) Reduces Voluntary Consumption of High Alcohol Concentrations in NonDependent Wistar Rats. Pharmacology Biochemistry and Behavior. 100(3):522-29. DOI: 10.1016/ j.pbb.2011.10.016

Collins, Michael A; Neafsey, Edward J; Mukamal, Kenneth J; Gray, Mary O; Parks, Dale A; Das, Dipak K; Korthuis, Ronald J. 2009; Alcohol in Moderation, Cardioprotection, and Neuroprotection: Epidemiological Considerations and Mechanistic Studies. Alcoholism: Clinical and Experimental Research. 33(2):206-19. DOI: 10.1111/j.1530-0277.2008.00828.x

Cordain, Loren; Eaton, S Boyd; Sebastian, Anthony; Mann, Neil; Lindeberg, Staffan; Watkins, Bruce A; O'Keefe, James H; Brand-Miller, Janette. 2005; Origins and Evolution of the Western Diet: Health Implications for the 21st Century. The American Journal of Clinical Nutrition. 81(2):34154. DOI: 10.1016/s0920-5861(96)00158-7 [PubMed: 15699220]

DeSantis TZ, Hugenholtz P, Larsen N, Rojas M, Brodie EL, Keller K, Huber T, Dalevi D, Hu P, Andersen GL. 2006; Greengenes, a Chimera-Checked 16S rRNA Gene Database and Workbench 
Compatible with ARB. Applied and Environmental Microbiology. 72(7):5069-72. DOI: 10.1128/ AEM.03006-05 [PubMed: 16820507]

Dietrich, Oliver; Manfred, Heun; Notroff, Jens; Schmidt, Klaus; Zarnkow, Martin. 2012; The Role of Cult and Feasting in the Emergence of Neolithic Communities. New Evidence from Gobekli Tepe, South-Eastern Turkey. Antiquity. 86(2012):674.doi: 10.1017/S0003598X00047840

Dwyer-Lindgren, Laura; Flaxman, Abraham D; Ng, Marie; Hansen, Gillian M; Murray, Christopher JL; Mokdad, Ali H. 2015; Drinking Patterns in US Counties from 2002 to 2012. American Journal of Public Health. 105(6):1120-27. DOI: 10.2105/AJPH.2014.302313 [PubMed: 25905846]

Eckburg, Paul B; Bik, Elisabeth M; Bernstein, Charles N; Purdom, Elizabeth; Dethlefsen, Les; Sargent, Michael; Gill, Steven R; Nelson, Karen E; Relman, David A. 2005; Diversity of the Human Intestinal Microbial Flora. Science (New York NY ). 308(5728):1635-38. DOI: 10.1126/ science. 1110591

Edelblum KL, Sharon G, Singh G, Odenwald MA, Sailer A, Cao S, Ravens S, Thomsen I, El Bissati K, McLeod R, Dong C, Gurbuxani S, Prinz I, Mazmanian SK, Turner JR. 2018; The Microbiome Activates CD4 T-cell mediated Immunity to Compensate for Increased Intestinal Permeability. Cell Mol Gastroenterol Hepatol. 4:285-297. DOI: 10.1016/j.jcmgh.2017.06.001

Edgar, Robert C. 2010; Search and Clustering Orders of Magnitude Faster than BLAST. Bioinformatics. 26(19):2460-61. DOI: 10.1093/bioinformatics/btq461 [PubMed: 20709691]

Engen, Phillip A; Green, Stefan J; Voigt, Robin M; Forsyth, Christopher B; Keshavarzian, Ali. 2015; The Gastrointestinal Microbiome: Alcohol Effects on the Composition of Intestinal Microbiota. Alcohol Research : Current Reviews. 37(2):223-36. DOI: 10.13140/RG.2.1.4342.9285 [PubMed: 26695747]

Fierer, Noah; Hamady, Micah; Lauber, Christian L; Knight, Rob. 2008; The Influence of Sex Handedness, and Washing on the Diversity of Hand Surface Bacteria. Proceedings of the National Academy of Sciences of the United States of America. 105(46):17994-99. DOI: 10.1073/pnas. 0807920105 [PubMed: 19004758]

Hartmann, Phillipp; Seebauer, Caroline T; Schnabl, Bernd. 2015; Alcoholic Liver Disease: The Gut Microbiome and Liver Cross Talk. Alcoholism: Clinical and Experimental Research. 39(5):76375. DOI: $10.1111 /$ acer.12704

Hold, Georgina L; Smith, Megan; Grange, Charlie; Watt, Euan Robert; El-Omar, Emad M; Mukhopadhya, Indrani. 2014; Role of the Gut Microbiota in Inflammatory Bowel Disease Pathogenesis: What Have We Learnt in the Past 10 Years? World Journal of Gastroenterology. 20(5):1192-1210. DOI: 10.3748/wjg.v20.i5.1192 [PubMed: 24574795]

Kelley, Scott T; Skarra, Danalea V; Rivera, Alissa J; Thackray, Varykina G. 2016; The Gut Microbiome Is Altered in a Letrozole-Induced Mouse Model of Polycystic Ovary Syndrome. PLoS ONE. 11(1)doi: 10.1371/journal.pone.0146509

Keshavarzian, Ali; Farhadi, Ashkan; Forsyth, Christopher B; Rangan, Jayanthi; Jakate, Shriram; Shaikh, Maliha; Banan, Ali; Fields, Jeremy Z. 2010; NIH Public Access. 50(3):538-47. DOI: 10.1016/j.jhep.2008.10.028.Evidence

Leclercq S, Matamoros S, Cani PD, Neyrinck AM, Jamar F, Starkel P, Windey K, et al. 2014; Intestinal Permeability, Gut-Bacterial Dysbiosis, and Behavioral Markers of Alcohol-Dependence Severity. Proc Natl Acad Sci U S A. 111(42):E4485-93. DOI: 10.1073/pnas.1415174111 [PubMed: 25288760]

Lozupone, Catherine; Knight, Rob. 2005; UniFrac: A New Phylogenetic Method for Comparing Microbial Communities. Applied and Environmental Microbiology. 71(12):8228-35. DOI: 10.1128/AEM.71.12.8228-8235.2005 [PubMed: 16332807]

McDonald, Daniel; Price, Morgan N; Goodrich, Julia; Nawrocki, Eric P; DeSantis, Todd Z; Probst, Alexander; Andersen, Gary L; Knight, Rob; Hugenholtz, Philip. 2012; An Improved Greengenes Taxonomy with Explicit Ranks for Ecological and Evolutionary Analyses of Bacteria and Archaea. The ISME Journal. 6(3):610-18. DOI: 10.1038/ismej.2011.139 [PubMed: 22134646]

Mulle, Jennifer G; Sharp, William G; Cubells, Joseph F. 2013; The Gut Microbiome: A New Frontier in Autism Research. Current Psychiatry Reports. 15(2)doi: 10.1007/s11920-012-0337-0

Mutlu, Ece a; Gillevet, Patrick M; Rangwala, Huzefa; Sikaroodi, Masoumeh; Naqvi, Ammar; Engen, Phillip a; Kwasny, Mary; Lau, Cynthia K; Keshavarzian, Ali. 2012; Colonic Microbiome Is 
Altered in Alcoholism. American Journal of Physiology. Gastrointestinal and Liver Physiology. 302(9):G966-78. DOI: 10.1152/ajpgi.00380.2011 [PubMed: 22241860]

Mutlu, Ece; Keshavarzian, Ali; Engen, Phillip; Forsyth, Christopher B; Sikaroodi, Masoumeh; Gillevet, Patrick. 2009; Intestinal Dysbiosis: A Possible Mechanism of Alcohol-Induced Endotoxemia and Alcoholic Steatohepatitis in Rats. Alcoholism: Clinical and Experimental Research. 33(10):1836-46. DOI: 10.1111/j.1530-0277.2009.01022.x

Nanji AA, Khettry U, Sadrzadeh SM. 1994; Lactobacillus feeding reduces endotoxemia and severity of experimental alcoholic liver (disease). Proc Soc Exp Biol Med. 205:243-7. [PubMed: 8171045]

National Institute on Alcohol Abuse and Alcoholism. [Accessed 18 January 2018] Drinking Levels Defined. 2018. Available at: https://www.niaaa.nih.gov/alcohol-health/overview-alcoholconsumption/moderate-binge-drinking

Queipo-Ortuño MI1, Boto-Ordóñez M, Murri M, Gomez-Zumaquero JM, Clemente-Postigo M, Estruch R, Cardona Diaz F, Andrés-Lacueva C, Tinahones FJ. 2012; Influence of red wine polyphenols and ethanol on the gut microbiota ecology and biochemical biomarkers. Am J Clin Nutr. 95:1323-34. DOI: 10.3945/ajcn.111.027847 [PubMed: 22552027]

Room, Robin; Babor, Thomas; Rehm, Jürgen. 2005; Alcohol and Public Health. Lancet. doi: 10.1016/ S0140-6736(05)17870-2

Schwabe, Robert F; Jobin, Christian. 2013; The Microbiome and Cancer. Nature Reviews. Cancer. 13(11):800-812. DOI: 10.1038/nrc3610 [PubMed: 24132111]

Segawa, Shuichi; Wakita, Yoshihisa; Hirata, Hiroshi; Watari, Junji. 2008; Oral Administration of HeatKilled Lactobacillus Brevis SBC8803 Ameliorates Alcoholic Liver Disease in Ethanol-Containing Diet-Fed C57BL/6N Mice. International Journal of Food Microbiology. 128(2):371-77. DOI: 10.1016/j.ijfoodmicro.2008.09.023 [PubMed: 18976829]

Simms, Jeffrey A; Steensland, Pia; Medina, Brian; Abernathy, Kenneth E; Chandler, L Judson; Wise, Roy; Bartlett, Selena E. 2008; Intermittent Access to 20\% Ethanol Induces High Ethanol Consumption in Long-Evans and Wistar Rats. Alcoholism: Clinical and Experimental Research. 32(10):1816-23. DOI: 10.1111/j.1530-0277.2008.00753.x

Stockwell, Tim; Zhao, Jinhui; Panwar, Sapna; Roemer, Audra; Naimi, Timothy; Chikritzhs, Tanya. 2016; Do 'Moderate' Drinkers Have Reduced Mortality Risk? A Systematic Review and MetaAnalysis of Alcohol Consumption and All-Cause Mortality. Journal of Studies on Alcohol and Drugs. 77(2):185-98. DOI: 10.15288/jsad.2016.77.185 [PubMed: 26997174]

Tilg H, Kaser A. 2011; Gut Microbiome, Obesity, and Metabolic Dysfunction. J Clin Invest. 121(6): 2126-32. DOI: 10.1172/JCI58109 [PubMed: 21633181]

Turnbaugh, Peter J; Ley, Ruth E; Mahowald, Michael A; Magrini, Vincent; Mardis, Elaine R; Gordon, Jeffrey I. 2006; An Obesity-Associated Gut Microbiome with Increased Capacity for Energy Harvest. Nature. 444(7122):1027-1131. DOI: 10.1038/nature05414 [PubMed: 17183312]

Wang, Qiong; Garrity, George M; Tiedje, James M; Cole, James R. 2007; Naïve Bayesian Classifier for Rapid Assignment of rRNA Sequences into the New Bacterial Taxonomy. Applied and Environmental Microbiology. 73(16):5261-67. DOI: 10.1128/AEM.00062-07 [PubMed: 17586664]

Wang Y, Kirpich I, Liu Y, Ma Z, Barve S, McClain CJ, Feng W. 2011; Lactobacillus rhamnosus GG treatment potentiates intestinal hypoxia-inducible factor, promotes intestinal integrity and ameliorates alcohol-induced liver injury. Am J Pathol. 179:2866-75. DOI: 10.1016/j.ajpath. 2011.08.039 [PubMed: 22093263]

Wang Y, Liu Y, Sidhu A, Ma Z, McClain C, Feng W. 2012; Lactobacillus rhamnosus GG culture supernatant ameliorates acute alcohol-induced intestinal permeability and liver injury. Am J Physiol Gastrointest Liver Physiol. 303:G32-41. DOI: 10.1152/ajpgi.00024.2012 [PubMed: 22538402]

Yan, Arthur W; Fouts, Derrick E; Brandl, Johannes; Stärkel, Peter; Torralba, Manolito; Schott, Eckart; Tsukamoto, Hide; Nelson, Karen E; Brenner, David a; Schnabl, Bernd. 2011; Enteric Dysbiosis Associated with a Mouse Model of Alcoholic Liver Disease. Hepatology. 53(1):96-105. DOI: 10.1002/hep.24018 [PubMed: 21254165] 
Yatsunenko, Tanya; Rey, Federico E; Manary, Mark J; Trehan, Indi; Dominguez-Bello, Maria Gloria; Contreras, Monica; Magris, Magda; , et al. 2012; Human Gut Microbiome Viewed across Age and Geography. Nature. doi: 10.1038/nature11053

Yu T, Guo F, Yu Y, Sun T, Ma D, Han J, Qian Y, Kryczek I, Sun D, Nagarsheth N, Chen Y, Chen H, Hong J, Zou W, Fang J-Y. 2017; Fusobacterium nucleatum Promotes Chemoresistance to

Colorectal Cancer by Modulating Autophagy. Cell. 170:548-563. e16. [PubMed: 28753429] 

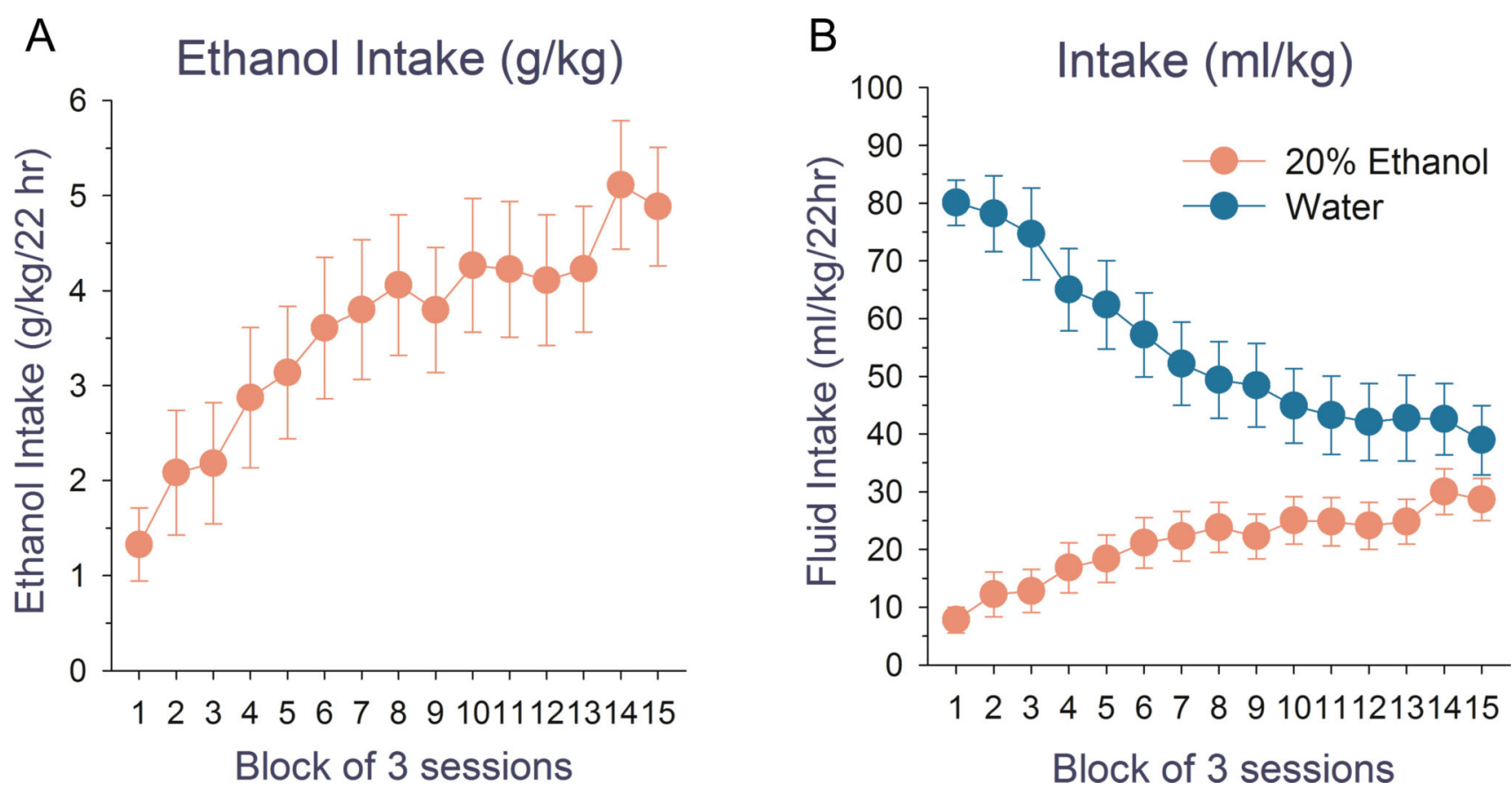

Figure 1. Ethanol consumption over the 3-month voluntary exposure period Mean $( \pm$ SE) $\mathrm{g} / \mathrm{kg}$ ethanol intake (A) and $\mathrm{ml} / \mathrm{kg}$ ethanol and water intake (B) in male Wistar rats across all 15 session blocks of ethanol exposure (45 sessions total) in the intermittentaccess $20 \%$ ethanol drinking paradigm. Individual session data were averaged in 3-session blocks prior to analysis. 

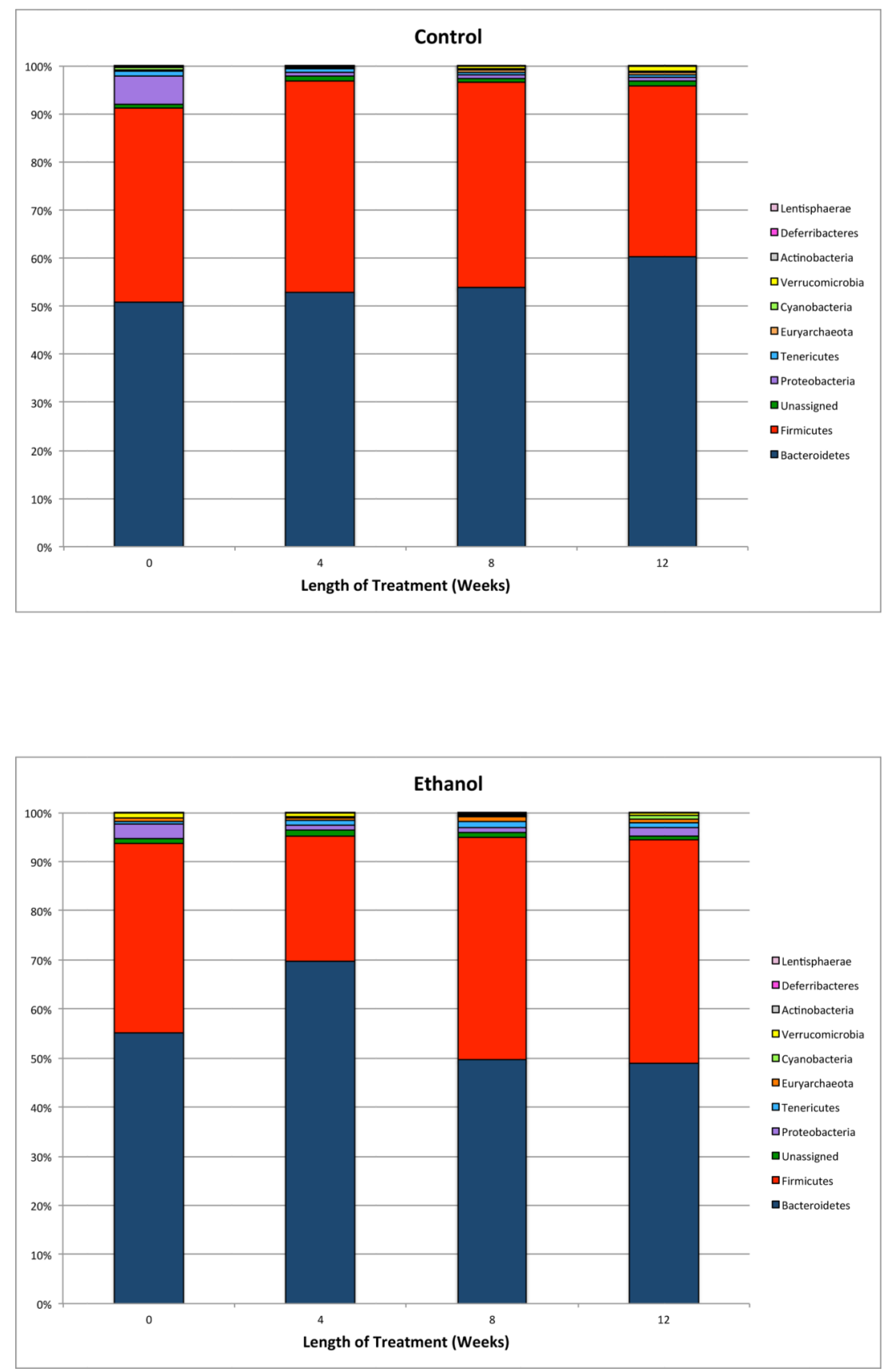

Figure 2. Ethanol treatment does not alter gut microbiota at the phylum level Bar charts illustrate abundances at baseline (time 0) and 4-week time points throughout the study in control and ethanol-treated rats. There were no significant shifts in OTUs in the microbiome of ethanol-treated rats. 

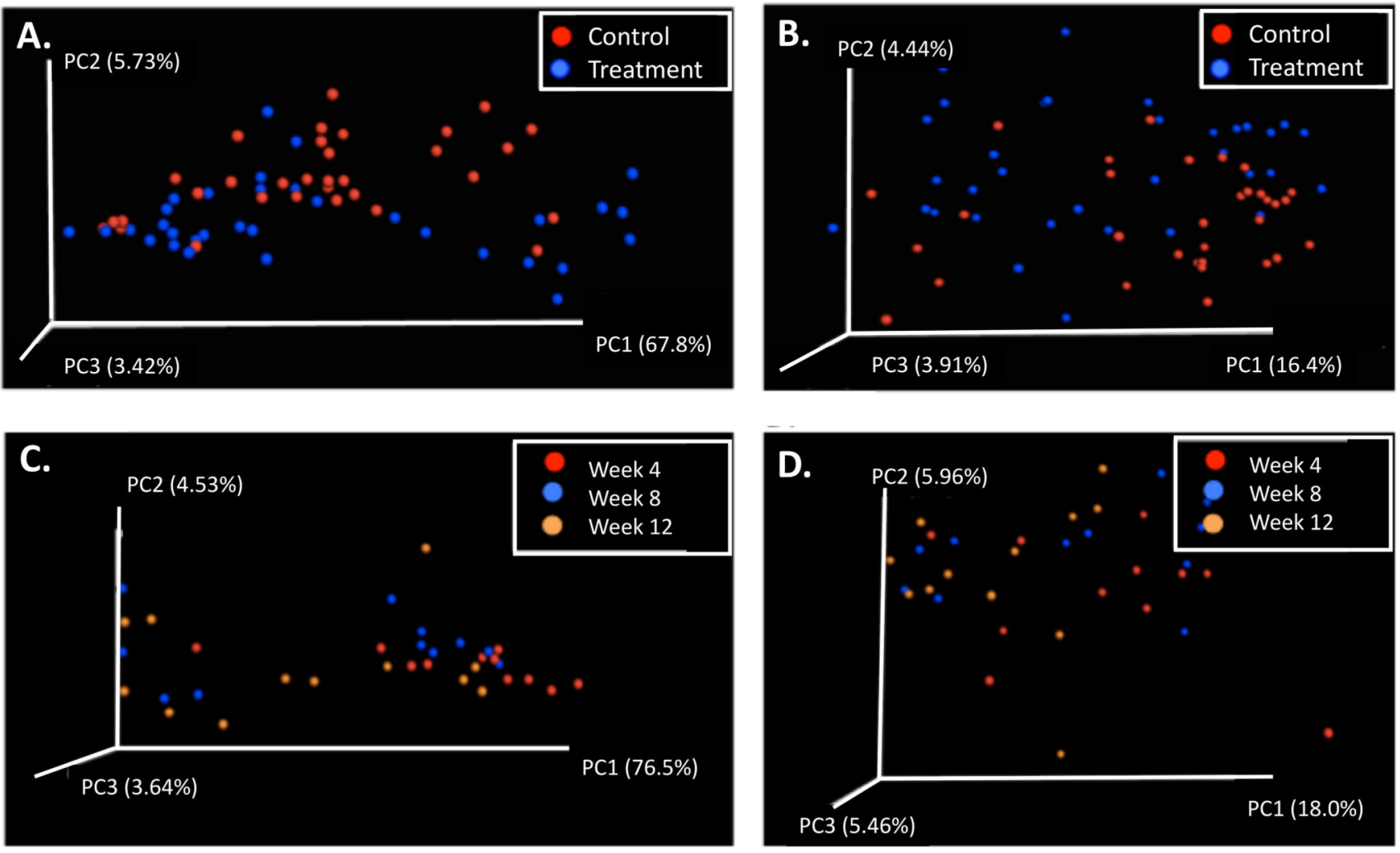

Figure 3. Ethanol treatment alters the gut microbiome

Shifts in gut microbiota were observed between treatments using weighted and unweighted UniFrac distances. Weighted UniFrac distances between control and ethanol groups (A), Unweighted UniFrac distances between control and ethanol groups (B). Gut microbiota were differentiated over time in ethanol-treated rats, but not in the control; Weighted Unifrac distances in the ethanol group over a 12-week period (C), Unweighted UniFrac distances in the ethanol group over a 12 -week period (D). 


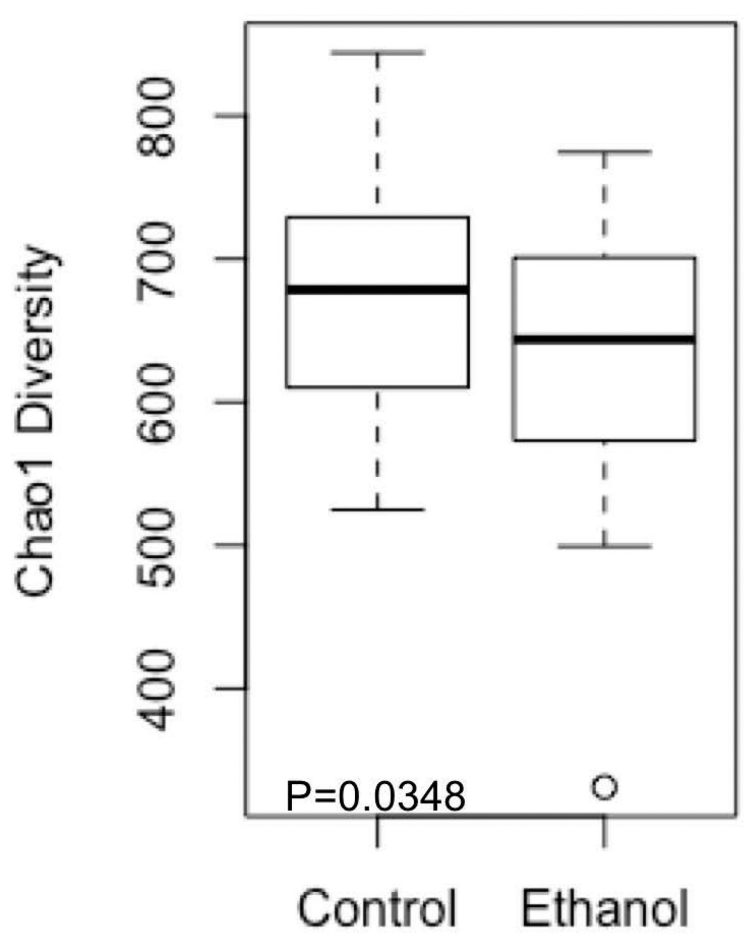

Treatment

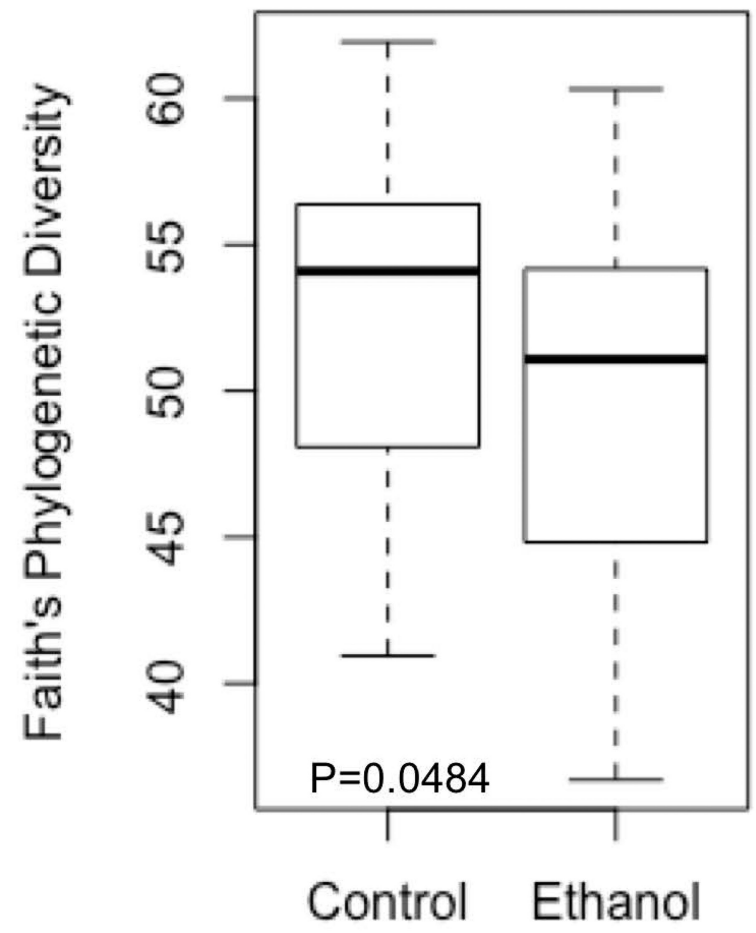

Treatment

Figure 4. Ethanol treatment results in a lower species richness and phylogenetic diversity Significant differences in alpha diversity, species richness (Chao1) and phylogenetic diversity (Faith's PD) determined between the control and ethanol groups. Overall, rats in the control group possessed greater diversity in the gut than those that consumed ethanol. 

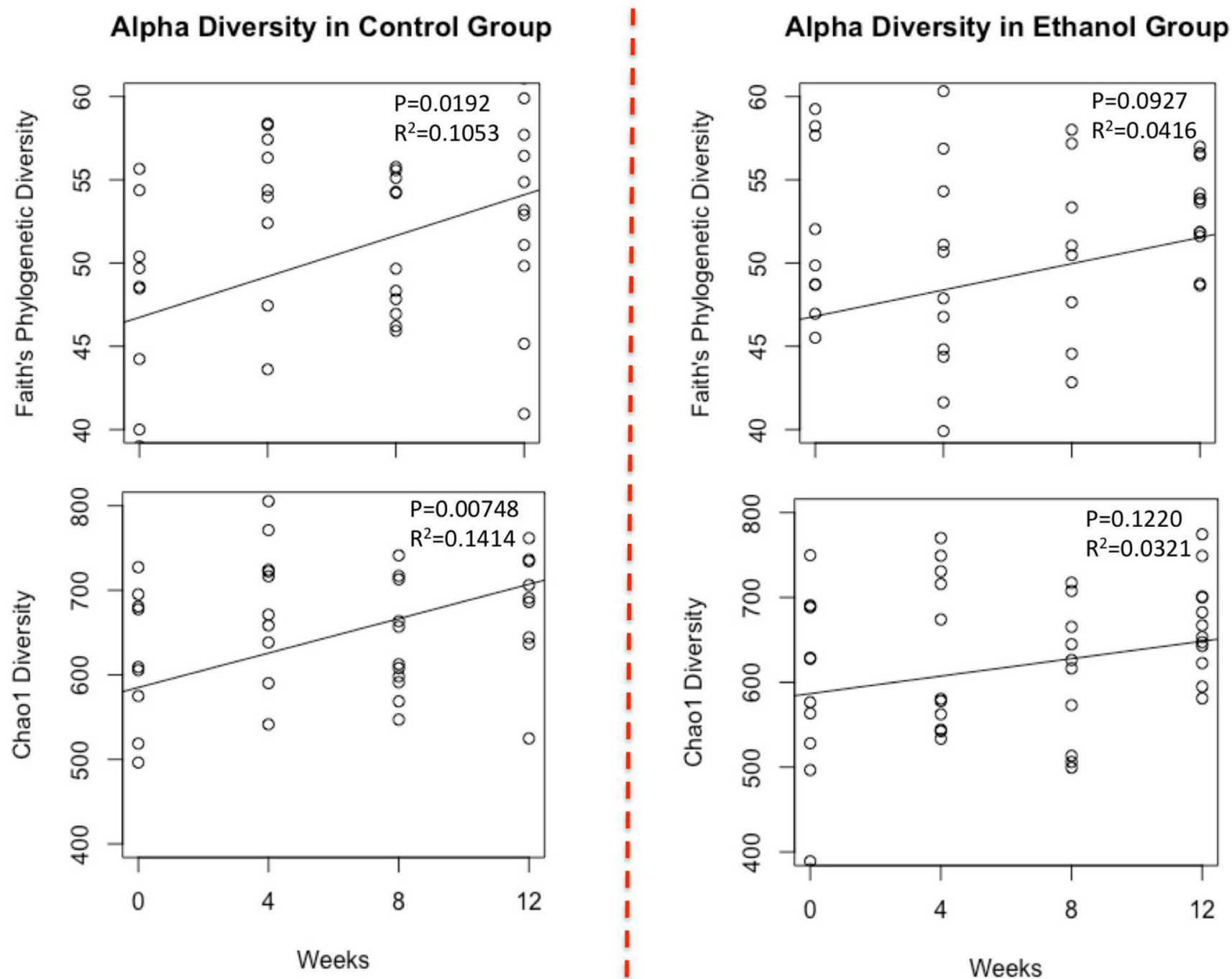

Figure 5. Effects of voluntary ethanol consumption on phylogenetic diversity and species richness over time

In the control group, there were significant increases in phylogenetic diversity (Faith's PD) and species richness (Chao1) over the four time points. In ethanol treated rats, the slope of the best fit line was not significantly different from zero $(\mathrm{P}>0.05)$. 
Lactobacillus (Firmicutes)
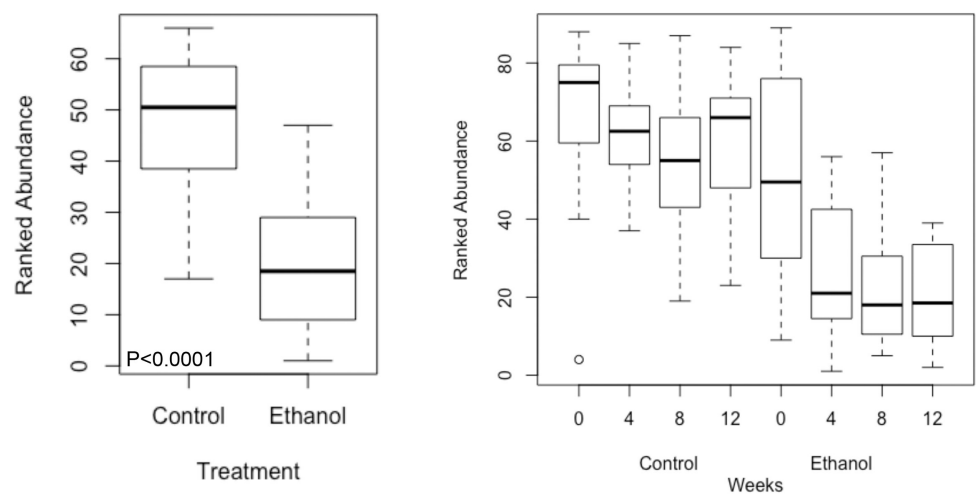

Peptostreptococcaceae (Firmicutes)
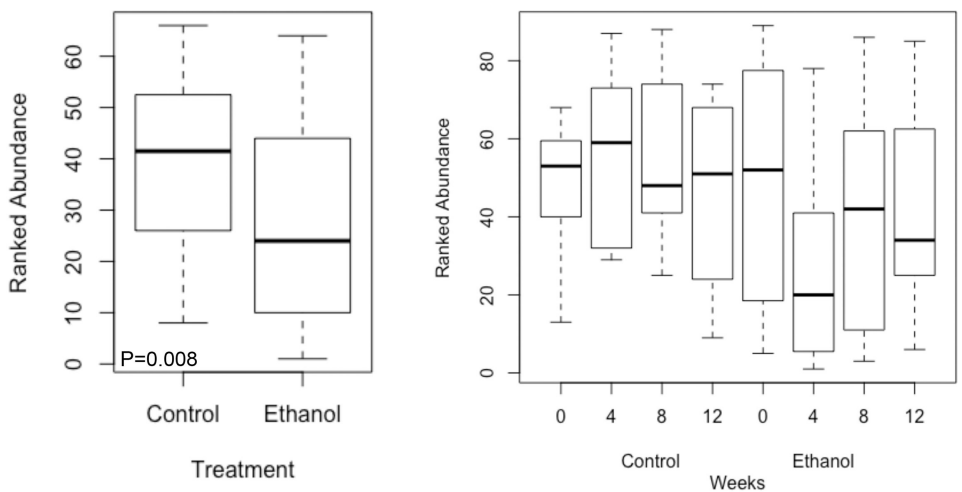

Turicibacter (Firmicutes)
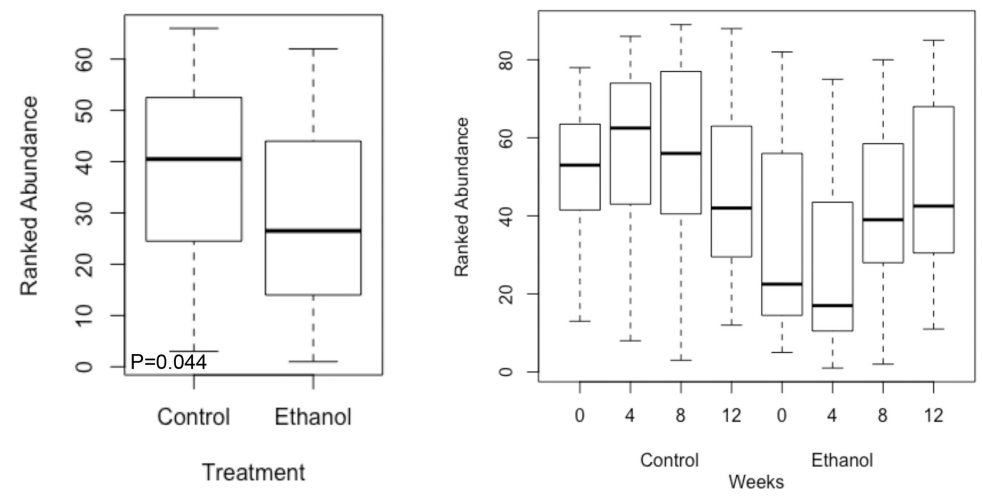

Figure 6. Select microbial taxa relative abundances that were lower in ethanol-treated rats Boxplots of mean ranked relative abundances of specific taxa by treatment over the course of the experiments not including Week 0 (left side) and at weekly time points (right side). Lactobacillus (Kruskal-Wallis test; $\mathrm{p}=4.9 \mathrm{E}-12$ ) abundances were significantly lower in ethanol-treated rats. Time-dependent response of Lactobacillus remained consistent in the control group, but decreased drastically from Week 0 to Week 4 and remained low in abundance in the ethanol group. We also observed significantly lower relative abundances of other Firmicutes, such as the Peptostreptococcaceae (K-W test; $\mathrm{p}=0.008)$ and Turicibacter 
(K-W test; $\mathrm{p}=0.044)$. No significant differences were found between the relative abundances of these taxa at Time 0 ; Table $\mathrm{S} 1$. 
Parabacteroides (Bacteroidetes)
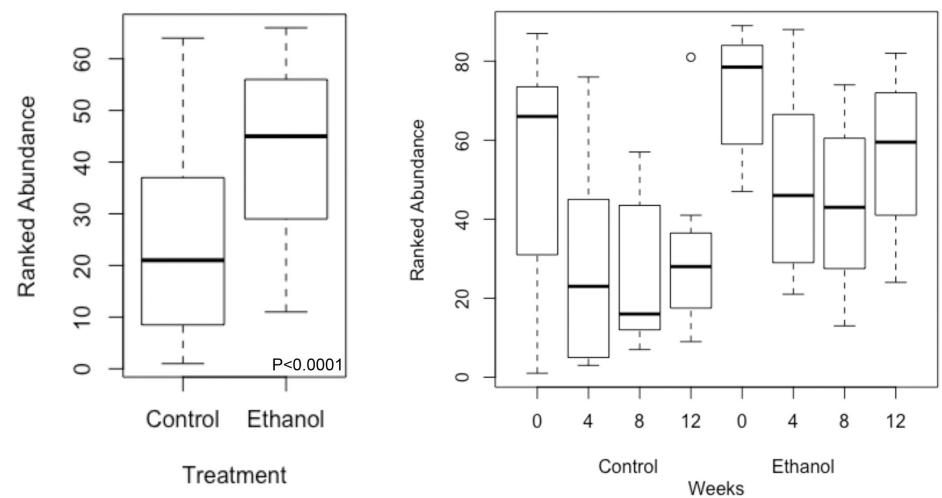

Barnesiellaceae (Bacteroidetes)
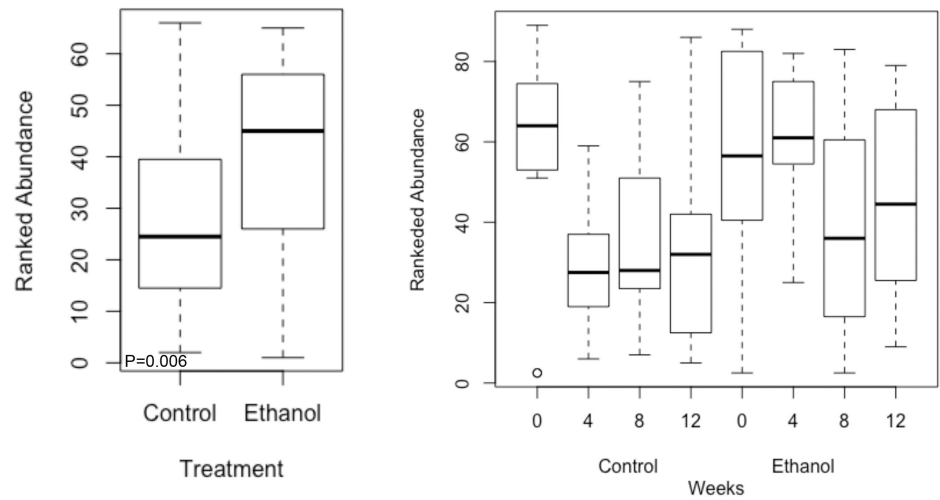

Bacteroides (Bacteroidetes)
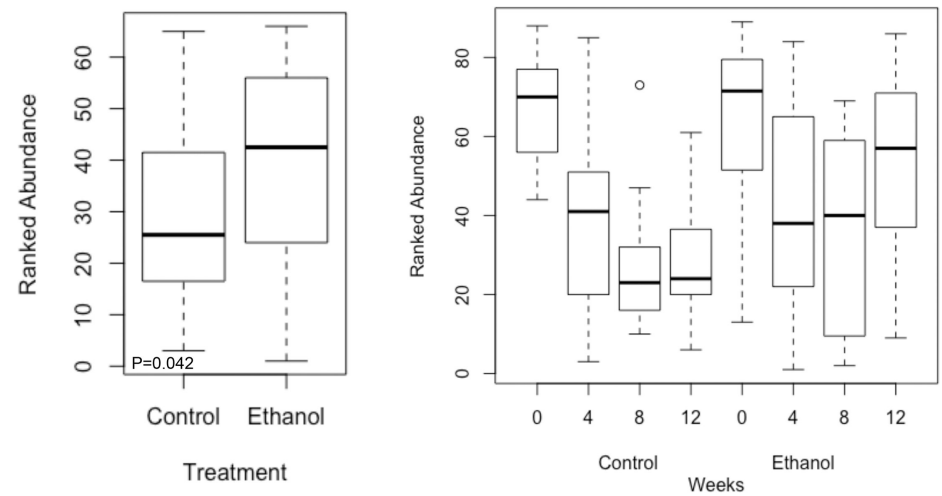

Figure 7. Select microbial taxa relative abundances that were higher in ethanol-treated rats Boxplots of mean ranked relative abundances of specific taxa by treatment over the course of the experiments not including Week 0 (left side) and at weekly time points (right side). The relative abundances of many Bacteroidetes, including Parabacteroides (K-W test; $\mathrm{p}=5.1 \mathrm{E}-05)$, Barnesiellaceae $(\mathrm{K}-\mathrm{W}$ test; $\mathrm{p}=0.006)$, and Bacteroides $(\mathrm{K}-\mathrm{W}$ test; $\mathrm{p}=0.042)$ were significantly higher in rats consuming ethanol than those belonging to the control group. No significant differences were found between the relative abundances of these taxa at Time 0; Table S1. 


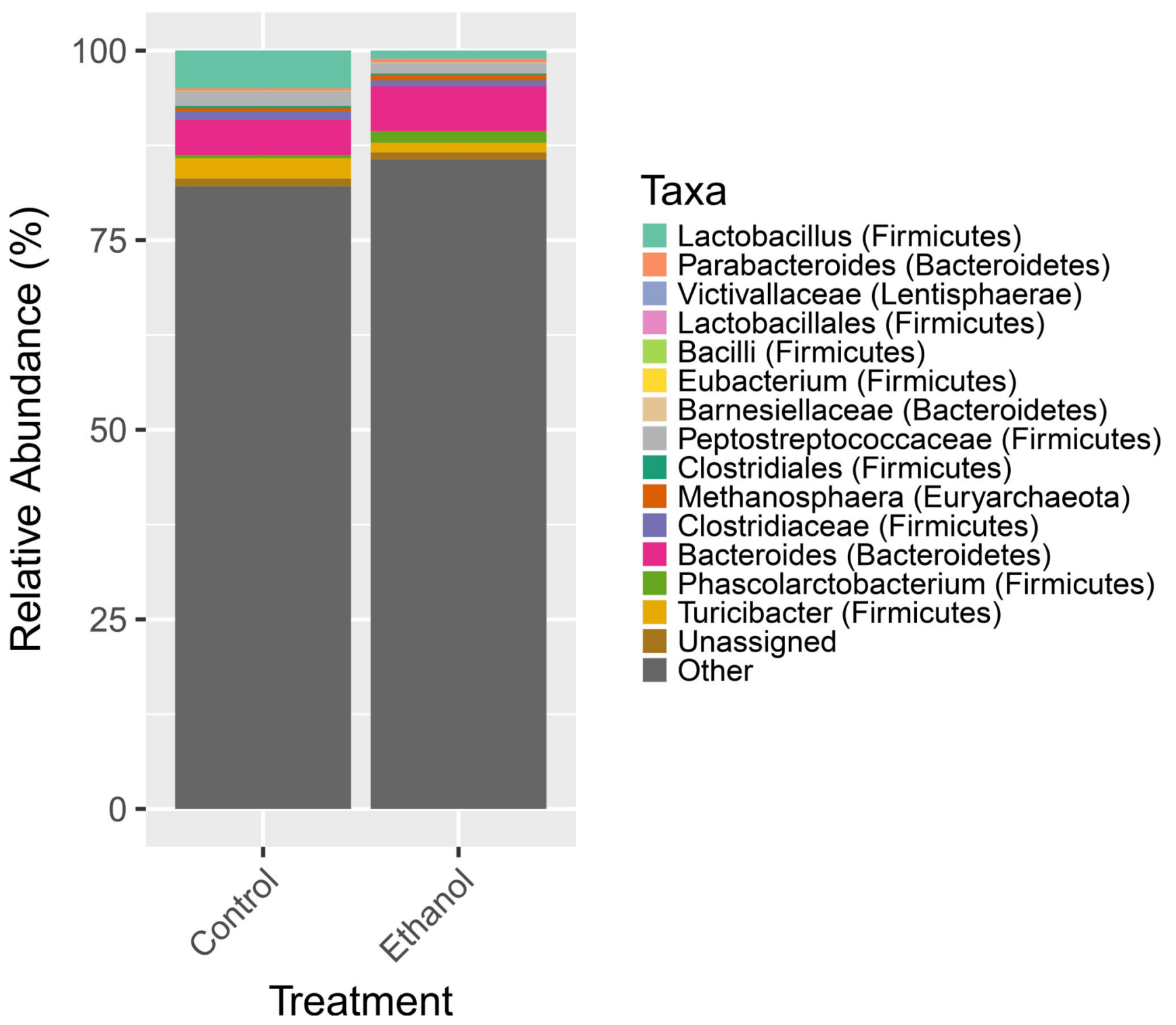

Figure 8. Ethanol treatment resulted in significant shifts at the genera level

The bar chart emphasizes the proportions of the 14 OTU abundances in the rat gut microbiome whose relative abundances differed significantly between treatments. Overall, those belonging to the phylum Firmicutes were lower and OTUs belonging to the phylum Bacteroidetes were higher in ethanol-treated rats. Methanosphaera, an archaeon belonging to the Euryarchaeota phylum, had higher abundance in ethanol-treated rats. 

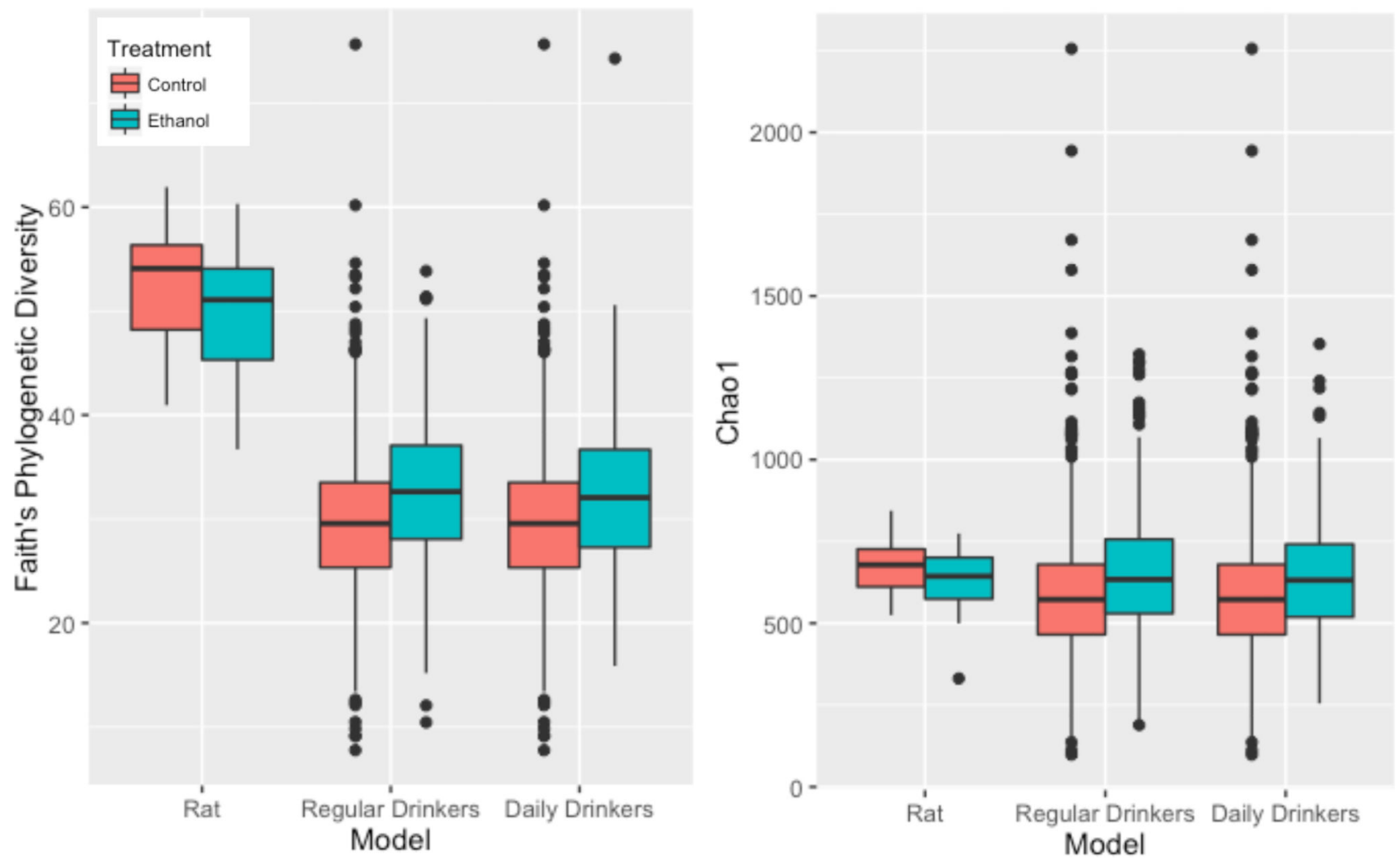

Figure 9. Higher Alpha Diversity in the Gut of Human Ethanol Consumers

While ethanol-treated rats displayed significantly lower species richness compared to controls, both groups of human drinkers displayed higher diversity in the gut relative to nondrinkers (Regular Drinkers: Faith's PD, p=2E-16, Chao1, p=5.34E-15; Daily Drinkers: Faith's PD, $\mathrm{p}=1.2 \mathrm{E}-12$, Chao1, $\mathrm{p}=1.47 \mathrm{E}-08$ ). While non-drinkers tended to be younger than regular and daily drinker (Table S2), an ANCOVA analysis found no significant interaction between age and frequency of reported alcohol consumption in terms of alpha diversity (Table S3). 


\section{Table 1}

Summary of significantly $(\mathrm{p}<0.05)$ different taxonomic relative abundances between treatment groups. (Mean abundances for each treatment group were summed over the course of the study excluding the initial time 0 collection.) Plus (+) sign indicates higher abundance in Ethanol Group vs. Control and minus (-) indicates lower abundance.

\begin{tabular}{|c|c|c|}
\hline OTU ( $97 \%$ similarity) & Rat $(\mathrm{N}=\mathbf{2 4})$ & Human $(\mathrm{N}=2401)^{a}$ \\
\hline \multicolumn{3}{|l|}{ Clustered to Genus } \\
\hline Parabacteroides & $+*$ & $+{ }^{*}$ \\
\hline Adlercreutzia & + & $+*$ \\
\hline Anaerostipes & + & $+*$ \\
\hline Barnesiellaceae & + & $+*$ \\
\hline Butyricimonas & + & $+*$ \\
\hline Clostridiales & + & $+*$ \\
\hline Oxalobacter & + & $+*$ \\
\hline Paraprevotella & + & $+{ }^{*}$ \\
\hline Phascolarctobacterium & + & $+*$ \\
\hline RF32 & + & $+^{*}$ \\
\hline Ruminococcaceae & + & $+*$ \\
\hline Sutterella & + & $+*$ \\
\hline S24-7 & + & $+{ }^{*}$ \\
\hline YS2 & + & $+{ }^{*}$ \\
\hline Coriobacteriaceae & - & $+*$ \\
\hline Dehalobacterium & - & $+*$ \\
\hline Peptococcus & - & - $^{*}$ \\
\hline Clostridiaceae & - & $-^{*}$ \\
\hline Lactobacillus & $-^{*}$ & - \\
\hline$c c 15$ & NA & $+*$ \\
\hline Eggerthella & NA & - $^{*}$ \\
\hline Lachnobacterium & NA & $+*$ \\
\hline Odoribacter & NA & $+*$ \\
\hline
\end{tabular}




\section{Table 2}

Comparisons of alpha diversity metrics determined for the rat data and the AGP data. Plus (+) sign indicates higher mean values summed over the course of the study (excluding time 0 ) in the ethanol group vs. control; minus (-) indicates the opposite.

\begin{tabular}{lccc}
\hline $\begin{array}{l}\text { Alpha Diversity } \\
\text { Measure }\end{array}$ & Rat $(\mathbf{N = 2 4 )}$ & $\begin{array}{c}\text { Regular Consumption } \\
(\mathbf{N = 2 7 5 5})\end{array}$ & $\begin{array}{c}\text { Daily Consumption } \\
(\mathbf{N = 2 1 8 7})\end{array}$ \\
\hline Chao1 & $-{ }^{*}$ & $+{ }^{*}$ & $+{ }^{*}$ \\
Faith's PD & $-{ }^{*}$ & $+{ }^{*}$ & $+{ }^{*}$ \\
Observed Species & $-{ }^{*}$ & $+{ }^{*}$ & $+{ }^{*}$ \\
Shannon & - & $+{ }^{*}$ & $+{ }^{*}$ \\
\hline indicates taxonomic abundances that were significantly (p<0.05) different treatment groups. \\
Regular Consumption refers to consumption of ethanol 3-5 times a week in humans. \\
Daily Consumption refers to daily consumption of ethanol in humans.
\end{tabular}

